Please do not remove this page

RMIT

UNIVERSITY

\title{
Small changes in particle-size distribution dramatically delay and enhance nucleation in hard sphere colloidal suspensions
}

Schoepe, Hans-Jaochim; Bryant, Gary; Van Megen, William

https://researchrepository.rmit.edu.au/esploro/outputs/9921862068501341/filesAndLinks?institution=61RMIT_INST\&index=null

Schoepe, H.-J., Bryant, G., \& Van Megen, W. (2006). Small changes in particle-size distribution dramatically delay and enhance nucleation in hard sphere colloidal suspensions. Physical Review E, 74(6), 1-4.

https://doi.org/10.1103/PhysRevE.74.060401

Published Version: https://doi.org/10.1103/PhysRevE.74.060401

Repository homepage: https://researchrepository.rmit.edu.au

(C)2006 The American Physical Society

Downloaded On 2023/04/26 21:12:21 +1000 


\title{
Small changes in particle-size distribution dramatically delay and enhance nucleation in hard sphere colloidal suspensions
}

\author{
Hans Joachim Schöpe, ${ }^{1}$ Gary Bryant, ${ }^{2}$ and William van Megen ${ }^{2}$ \\ ${ }^{1}$ Institut für Physik, Johannes Gutenberg-Universität Mainz, Staudingerweg 7, D-55128 Mainz, Germany \\ ${ }^{2}$ Department of Applied Physics, RMIT, GPO Box, 2476V, Melbourne 3001, Australia
}

(Received 4 September 2006; published 4 December 2006)

\begin{abstract}
We present hard-sphere crystallization kinetics for three samples with small differences in polydispersity. We show that an increase in polydispersity of $1 \%$ is sufficient to cause dramatic changes in the crystallization kinetics: Crystallization is delayed by almost one decade in time and quantitative and qualitative changes in the crystallization scenario are observed. Surprisingly the nucleation rate density is enhanced by almost a factor of 10 . We interpret these results in terms of polydispersity limited growth, where local fractionation processes lead to a delayed but faster nucleation.
\end{abstract}

DOI: 10.1103/PhysRevE.74.060401

PACS number(s): 82.70.Dd, 64.70.Dv, 81.10.Fq

Hard sphere colloidal suspensions are an important model system for the experimental study of crystallization and the glass transition [1]. The time scales of both the dynamics and kinetics of crystallization are accessible using light scattering techniques $[2,3]$. This is partly due to their large size (hundreds of $\mathrm{nm}$ ), and the Brownian nature of their motion. However, an important contributor to the slowing of crystallization is the fact that colloidal particles are always polydisperse. This polydispersity, fortuitously, allows the detailed study of crystallization in real time; however, it also complicates the interpretation of crystallization measurements, something which is only now being fully appreciated [4-6].

Experimental studies of the effects of polydispersity on crystallization have been limited to comparing different particles with different particle size distributions (PSDs)[4,7], or creating bidisperse mixtures of particles with (slightly) different particle sizes $[8,9]$. Theoretical work is even more scarce, and has largely concentrated on the equilibrium phase diagram $[10,11]$. The only study of the effects of polydispersity on crystallization kinetics is a computer simulation study of nucleation kinetics in hard spheres as a function of polydispersity [12], where it was found that crystal nucleation is suppressed by polydispersity.

We recently demonstrated [6] that the nucleation process in colloidal hard spheres occurs in two stages. The first, precursor stage, is characterized by the growth of clusters with long-lived nonequilibrium structures. The second stage is the delayed, primary nucleation process. We suggested that polydispersity limited growth is responsible for the precursor stage, and leads directly to the delay in primary nucleation.

Experiments with binary colloidal hard spheres [9] showed that the PSD has an influence on many parameters such as the induction time, average crystal size, crystal growth velocity, crystal conversion rate, and the equilibrium phase diagram. In addition, the crystal structure factor is affected which makes a quantitative analysis more difficult.

However, to date no experiments have systematically studied nucleation kinetics as a function of the polydispersity. In addition, nothing is known about how polydispersity in a one component system affects the time trace or magnitude of the nucleation rate density during crystallization, nor about the influence polydispersity has on crystal morphology.

In this paper, we present the first systematic study of the effects of small, controlled changes in the PSD in a one component system on the crystallization kinetics of hard spheres to obtain a comprehensive picture of the crystallization process. We present measurements for identical particles with three different, carefully characterized, polydispersities in the range of $4.8 \%$ to $5.8 \%$. These small changes in the PSD allow us to compare the time dependent crystalline structure factors. From these, we investigate the effect of polydispersity on crystallinity, crystal volume fraction, characteristic times, crystal sizes, and nucleation rates. We find that increasing the polydispersity by $\sim 1 \%$ leads to dramatic qualitative and quantitative changes in the crystallization scenario. Specifically, crystallization is significantly delayed, but nucleation is enhanced, with consequent changes to the crystal morphology.

The experimental methods and analysis used here are described elsewhere $[6,7]$. The particles are a copolymer core of methylmethacrylate and trifluoroethylacrylate stabilized by an $\sim 10 \mathrm{~nm}$ thick layer of poly-12-hydroxystearic acid, and are suspended in cis-decalin. Full details of the fractionation and characterization process will be published elsewhere [13]. Briefly, the original stock suspension (XL65-A) was fractionated in two steps using centrifugation techniques, yielding two new samples XL65-B and XL65-C with different PSDs. Figure 1 shows the PSDs determined by electron microscopy of about 1000 particles for each sample. Starting with a skewed distribution with a polydispersity of $5.8 \%$ (XL65-A), in the two fractionation steps the width and the skewness of the distribution is reduced leading to a skewed distribution with $5.3 \%$ (XL65-B) and finally to an almost symmetric distribution with $4.8 \%$ (XL65-C). The polydispersities, defined as $\sigma(a) /\langle a\rangle$, were confirmed using static and dynamic light scattering. A detailed comparison of particle sizing using electron microscopy, form factor analysis, and dynamic light scattering will be the topic of a forthcoming article. To our knowledge, there is no study in which the PSD of one component hard sphere systems have been characterized in such a comprehensive way. The hydrodynamic radius is $R_{H}=(320 \pm 10) \mathrm{nm}$ which leads to a Brownian time $t_{B}=R^{2} / D_{0}=0.5 \mathrm{~s}$, where $D_{0}$ is the free particle diffusion coefficient. 


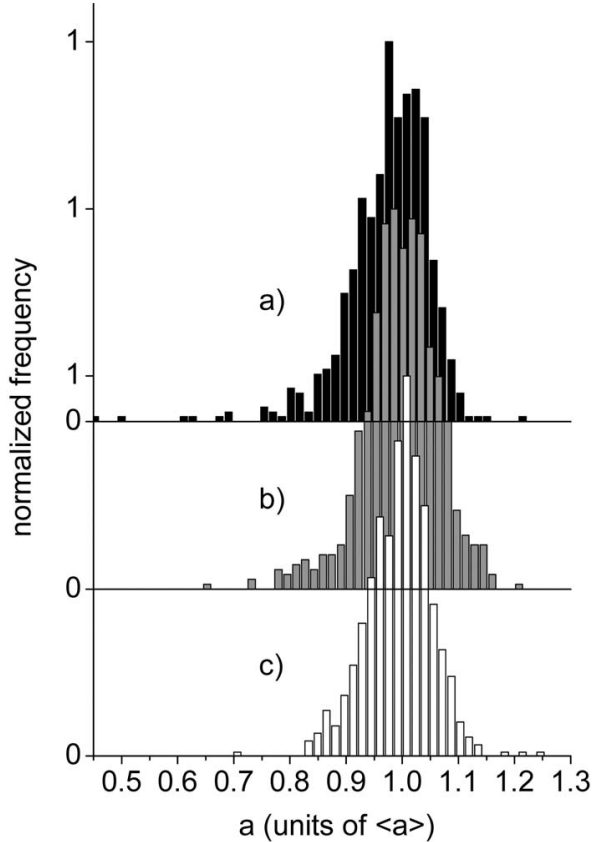

FIG. 1. PSDs of the three fractions used. (a) XL65-A, $\sigma=5.8 \%$, skewed; (b) XL65-B, $\sigma=5.3 \%$, skewed; (c) XL65-C, $\sigma=4.8 \%$, symmetric.

The particles behave as near perfect hard spheres [1]. The effective hard sphere volume fraction was determined by comparing the equilibrium phase diagram of the least polydisperse sample, XL65-C, to the known freezing volume fraction of hard spheres with a $5 \%$ polydispersity [10], giving a freezing point of 0.505 and a melting point of about 0.538. This scaling factor was used for all three samples. With regard to the experimental uncertainties we find the same freezing and melting volume fractions for the three fractions, which is in agreement with the simulations of Bolhuis and Kofke when varying the polydispersity between $4.8 \%$ and $5.8 \%$.

Crystallization kinetics were measured using time resolved laser light Bragg scattering averaging over the whole Debye-Scherrer cone to allow access to data at early times [14]. The angular resolution of the detector is $\Delta \theta=0.02^{\circ}$, giving a resolution around the main peak of about $\Delta q \approx 5$ $\times 10^{-6} \mathrm{~nm}^{-1}$. Scans can be done in time intervals of $60 \mathrm{~s}$. To our knowledge, there is no other experimental setup monitoring crystallisation kinetics in colloidal model systems providing a comparable performance. Crystal growth is characterized from the structure factor $S(q, t)$, as a function of the scattering vector $q$ and elapsed time $t$. The crystalline structure factor $S_{\text {xtal }}(q, t)=S(q, t)-\beta(t) S_{\text {fluid }}(q, t)$ is obtained by subtracting the fluid background $\beta(t) S_{\text {fluid }}$, which is measured $60 \mathrm{~s}$ after stopping the shear melting process [15].

From the crystalline structure factor, we extract: (i) The crystallinity, $X(t)=c \int S_{\text {xtal }}(q, t) d q$, determined by integrating over the area of the main Bragg reflection, normalized by the parameter $c$ obtained from the equilibrium phase diagram; (ii) the crystalline volume fraction $\phi_{x t a l}(t)$ $=2\left[q_{\max }(t) R\right]^{3} / 9 \sqrt{3} \pi^{2}$, determined from the peak maximum, $q_{\max }(t)$; (iii) the average linear dimension of the crystals,

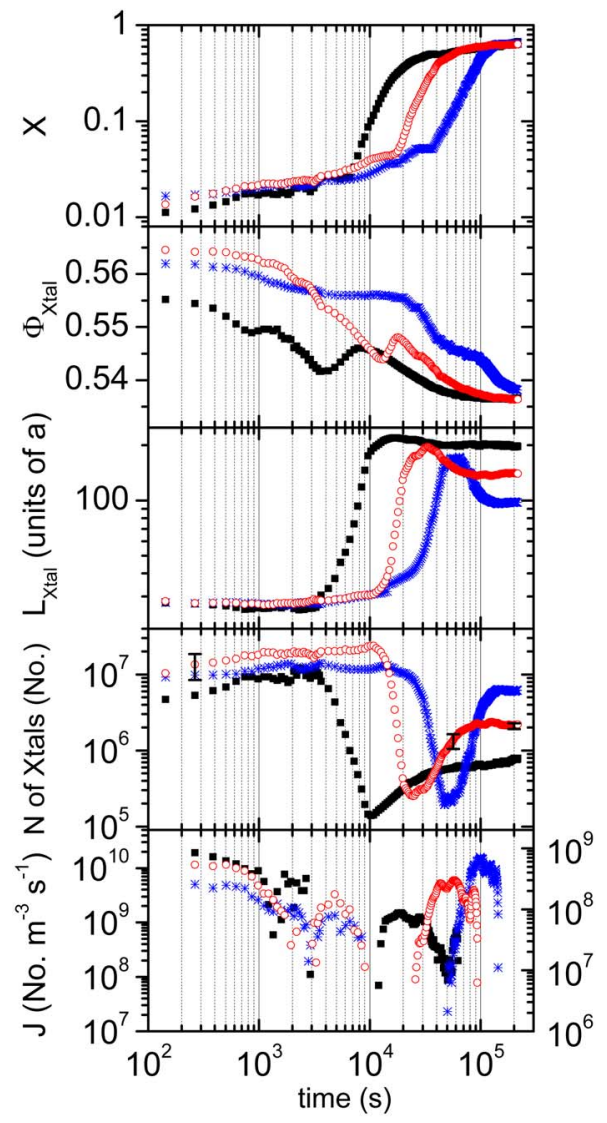

FIG. 2. (Color online) Crystallization parameters for $\Phi=0.527$ : Filled blue crosses XL65-A; open red circles XL65-B; filled black squares XL65-C. From top to bottom: Crystallinity, crystalline volume fraction, average linear dimension, absolute number of crystals, and nucleation rate densities.

$\langle L(t)\rangle=2 \pi K / \delta q(t)$, determined from $\delta q(t)$, the full width at half maximum of the peak, where $K=1.0747$ is the Scherrer constant for a spherical crystal [16]; (iv) the number of crystals in the observed scattering volume: $N_{\text {xtal }}(t)$ $=X(t) V_{\text {scatt }} /\left\langle L^{3}(t)\right\rangle=X(t) V_{\text {scatt }} / \alpha\langle L(t)\rangle^{3}$, where $V_{\text {scatt }}$ is the volume illuminated by the laser, and $\alpha \approx 1.25$ [17] connects the average crystal size cubed with the average crystal volume; and (v) the nucleation rate density, defined as the rate at which crystals appear in the liquid free volume:

$$
J(t)=\frac{1}{V_{\text {free }}(t)} \frac{d}{d t} N_{\text {xtal }}(t)=\frac{1}{[1-X(t)]} \frac{d}{d t} \frac{X(t)}{\left\langle L^{3}(t)\right\rangle} .
$$

Figure 2 shows the time dependent behavior of these five parameters extracted from the crystalline structure factors for each of the three samples in coexistence at a volume fraction of 0.527 . The first point to note is that relatively small differences in polydispersity (Fig. 1) lead to dramatic differences in the kinetics. For example, the main nucleation event is delayed by almost an order of magnitude with a $1 \%$ increase in polydispersity.

Second, all three samples exhibit a two step nucleation process, consistent with previous observations [6] - from the earliest times when deviations from the fluid structure factor 
can be observed, the amount of solid material $(X)$ grows slowly while the size of the clusters $\left(L_{\mathrm{xtal}}\right)$ remains nearly constant. The number of the crystalline precursors $(N)$ in the sample is increasing slowly, but there is limited growth of these precursors. As a result the nucleation rate density $(J)$ is slowly decreasing at early times. It is noticeable that the short time behavior is similar for the three samples, with no significant qualitative differences being apparent.

The following characteristics are common to all samples. The average crystal size rises sharply prior to the onset of the main nucleation process, and approaches a maximum while the crystallinity $(X)$ remains low-i.e., only a small part of the sample has crystallized. During this period the number of crystals $(N)$ declines sharply. In this region where $N$ is decreasing, the nucleation rate density $(J)$ is undefined. The remainder of the growth is characterized by rapid growth in the crystallinity $(X)$, accompanied by a tenfold increase in the number of crystals. In this main nucleation region the nucleation rate density shows a significant increase before reaching a maximum and decreasing to zero again. The nucleation rate density of the main nucleation process is always smaller than the nucleation rate density of the precursor nucleation. Finally, when the main nucleation process has ended, all parameters (except $J$ ) plateau and no significant ripening is evident on the time scale of these experiments. After crystallization is complete, all samples have the same crystal volume fraction, as determined from the fcc (111) peak position.

Despite the similarities between the samples, there are also distinct differences. The main nucleation process is delayed significantly for the more polydisperse samples, suggesting that this process is triggered by the local fractionation processes occurring since the quench. This is highlighted by the behavior of the average crystal size, which drops by almost a factor of 2 for the most polydisperse sample during the later stages of the nucleation process. This effect decreases as polydispersity is reduced. It should be noted that this decrease in the average crystal size is accompanied by a sudden increase in the number $(N)$ of small crystals, rather than by a reduction in size of the existing crystals.

Towards the end of the precursor stage, and at the beginning of the main nucleation phase, the crystal volume fraction exhibits nonmonotonic behavior. We interpret this as being due to the structure of the crystallites changing from early stage precursor structures into a random hexagonal closed packed structure, as described previously [6]. This is caused by local fractionation, so the average particle size inside the crystalline regions is changing. As polydispersity is increased the features of the volume fraction curve become less pronounced, and are significantly delayed, as more fractionation is required.

The differences in the time trace of the main nucleation process are highlighted in the linear-log representation shown in Fig. 3. At low polydispersity the nucleation rate density has a low, pronounced plateau, indicating a region where the nucleation rate is constant. This plateau is not seen at the higher polydispersities, where the nucleation rate is more peaked. Significantly, the nucleation process of the

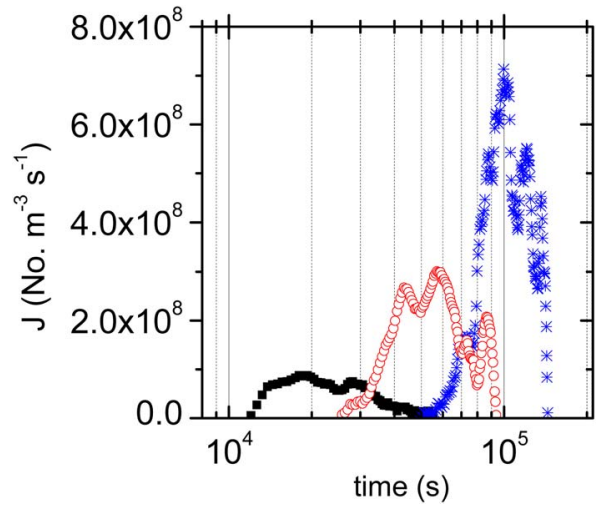

FIG. 3. (Color online) Nucleation rate densities of the main nucleation process $\Phi=0.527$ : Filled blue crosses XL65-A; open red circles XL65-B; filled black squares XL65-C.

most polydisperse system is delayed by an order of magnitude, but the maximum in the nucleation rate density is almost an order of magnitude higher. As can be seen in the time trace of the number of crystals $N$ and from the error bars in Fig. 2, these observations are statistically significant and are not caused by numerical differentiation artifacts. One might argue that the undercoolings of the three samples could be different-however, to obtain an increase in the nucleation rate density of one order of magnitude in the coexistence region requires an increase of at least $0.6 \%$ in volume fraction $[6,18]$ which is larger than the error in determining the volume fraction. The fact that we see a systematic increase with polydispersity negates this argument. The results obtained are in sharp contrast to Monte Carlo simulations $[12,19]$, which imply that increasing polydispersity leads to a reduction in the nucleation rate. They found that the computed free-energy barrier for crystal nucleation increases significantly by increasing the polydispersity at the same undercooling. The absolute nucleation rate densities at volume fractions around 0.535 show a more than tenfold increase for a monodisperse system compared to a system with $5 \%$ polydispersity.

It is clear from Figs. 2 and 3 that there are significant differences between the three samples. In order to begin to quantify the effects of polydispersity, in Fig. 4 we extract some characteristic quantities, and plot them as a function of polydispersity. Clearly we do not have a broad enough range of polydispersities to make quantitative extrapolations, but the trends observed are instructive.

The top panel shows the characteristic times derived from the crystallinity: The induction time, determined from the first crystalline features in the structure factor [6], and the crossing time where crystallization stops [15]. Both characteristic times show an increase of almost an order of magnitude as polydispersity increases-i.e., unsurprisingly the least polydisperse suspension is much less delayed.

The second panel shows the maximum and final crystal size, which both decrease significantly as polydispersity increases. Clearly the maximum crystal size is limited by polydispersity. The difference between the maximum and the final crystal size also increases with increasing polydispersity. For the higher polydispersities, a larger number of smaller 


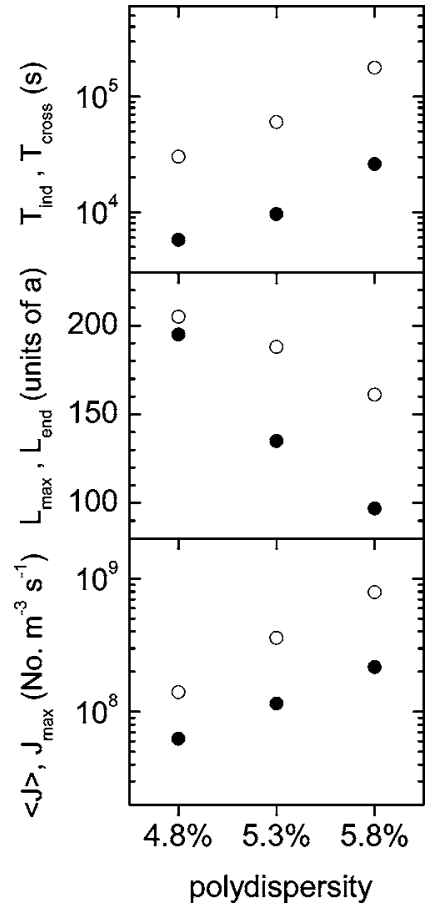

FIG. 4. From top to bottom: - Induction time (closed circles) and crossing time (open circles), maximum crystal size (open circles) and final crystal size (closed circles), and average nucleation rate density (closed circles) and maximum nucleation rate density (open circles) as functions of polydispersity $\Phi=0.527$.

crystals are created at the end of the crystallization process, thus leading to the large difference between maximum and final average crystal sizes. Interestingly, the final and maximum crystal sizes extrapolate to zero at around $7 \%$ and $10 \%$ polydispersity, respectively, consistent with most estimates of the limiting polydispersity for crystallization $[11,12,20,21]$. While interesting, clearly this is an oversimplification, as the concept of a limiting polydispersity is complicated by the extensive local fractionation which occurs during crystallization.

The third panel shows the average and maximum nucle- ation rate densities for the main nucleation process. Interestingly, although polydispersity significantly delays nucleation, when it finally does occur, it occurs more quickly for the more polydisperse samples-i.e., the nucleation rate increases with polydispersity. It appears that the delay in nucleation and the slowing down of crystal growth caused by polydispersity leads eventually to an extremely fast final crystallization. We interpret this as being due to the slow rearrangement and local fractionation of particles in the fluid. As polydispersity increases, this local fractionation takes longer and is more local, so when nucleation finally does proceed, the higher polydispersities produce a large number of smaller crystals, leading to a higher nucleation rate.

The results presented in this Rapid Communication confirm our previous suggestion [6] that the length of the induction process and the delay time prior to nucleation are approximately linearly related to the polydispersity. This delay is due to local fractionation of different sized particles, and contributes to the observed increase in the nucleation rate density with increasing polydispersity when nucleation finally occurs. This fractionation also ensures that when nucleation does occur, it occurs in many places, producing a larger number of smaller crystallites as polydispersity increases. Thus for high polydispersities the average crystal size shows a distinct temporal maximum, but the final average crystal size is lower.

We conclude by noting that, previously, studies on hard sphere crystallization have focused on volume fraction as the only parameter. This work conclusively shows that polydispersity is equally critical in colloidal hard spheres, and it is vital that comparisons between the results of different groups, or between different techniques, take polydispersity into account. Moreover, polydispersity can now be considered as an adjustable parameter, which may enable the optimization of crystallization parameters for a wide range of applications, as well as being a tool for better understanding the fundamentals of crystallization.

H.J.S. would like to thank the Alexander von Humboldt Foundation for providing financial support.
[1] P. N. Pusey and W. van Megen, Nature (London) 320, 340 (1986).

[2] W. van Megen and S. M. Underwood, Phys. Rev. E 47, 248 (1993).

[3] J. K. G. Dhont, C. Smits, and H. N. W. Lekkerkerker, J. Colloid Interface Sci. 152, 386 (1992).

[4] S. I. Henderson et al., Physica A 233, 102 (1996).

[5] S. Martin, G. Bryant, and W. van Megen, Phys. Rev. Lett. 90, 255702 (2003).

[6] H. J. Schöpe, G. Bryant, and W. van Megen, Phys. Rev. Lett. 96, 175701 (2006).

[7] S. Martin, G. Bryant, and W. van Megen, Phys. Rev. E 67, 061405 (2003).

[8] S. I. Henderson and W. van Megen, Phys. Rev. Lett. 80, 877 (1998).

[9] S. Martin, G. Bryant, and W. van Megen, Phys. Rev. E 71,
021404 (2005).

[10] D. A. Kofke and P. G. Bolhuis, Phys. Rev. E 59, 618 (1999).

[11] P. Bartlett, J. Phys.: Condens. Matter 12, A275 (2000).

[12] S. Auer and D. Frenkel, Nature (London) 413, 711 (2001).

[13] H. J. Schöpe, O. Marnette, and G. Bryant (unpublished).

[14] P. Francis et al., Rev. Sci. Instrum. 73, 3872 (2002).

[15] J. L. Harland and W. van Megen, Phys. Rev. E 55, 3054 (1997).

[16] A. L. Patterson, Phys. Rev. 56, 978 (1939).

[17] K. M. Dobrich, C. Rau, and C. E. I. Krill, Metall. Mater. Trans. A 35A, 1953 (2004).

[18] J. L. Harland et al., Phys. Rev. Lett. 75, 3572 (1995).

[19] S. Auer and D. Frenkel, J. Chem. Phys. 120, 3015 (2004).

[20] P. N. Pusey, J. Phys. (Paris) 48, 709 (1987).

[21] M. Fasolo and P. Sollich, Phys. Rev. E 70, 041410 (2004). 